\title{
LA MIGRACIÓN HACIA SOFTWARE LIBRE EN LA FORMACIÓN DE PROFESORES DE INFORMÁTICA COMO ESTRATEGIA DE SOBERANÍA TECNOLÓGICA
}

\author{
A MIGRAÇÃO PARA O SOFTWARE LIVRE NA FORMAÇ̃̃O DE PROFESSORES DE \\ INFORMÁTICA COMO ESTRATÉGIA DE SOBERANIA TECNOLÓGICA
}

\author{
MIGRATION TOWARDS FREE SOFTWARE IN THE TRAINING OF COMPUTER \\ TEACHERS AS A STRATEGY OF TECHNOLOGICAL SOVEREIGNTY
}

Walfredo González Hernández

Doctor en Ciencias Pedagógicas

Universidad de Matanzas - UM

Matanzas, Matanzas - Cuba. walfredo.glez@umcc.cu

Dayana Olivia Hernández Revilla
Máster en Administración de Empresas
Universidad de Matanzas - UM.
Matanzas, Matanzas - Cuba.
dayana.hernandez@ umcc.cu

Juan Manuel González Castillo
Doctor en Ciencias Pedagógicas
Universidad de Matanzas - UM.
Matanzas, Matanzas - Cuba.
juanmanuel.castillo@ umcc.cu

Resumen: Se analiza la concepción de la soberanía tecnológica para el tratamiento de los sistemas que se van a implantar en el país y las decisiones sobre su modificación. Sin embargo, este es un proceso complejo para su implementación, si no se logra el desarrollo de competencias informacionales en los profesores de informática, que tomen como base el software libre. El análisis de la formación de estos profesionales debe partir de la estructuración de un diseño curricular con asignaturas propias y optativas que se estructuren en función de los objetivos del proceso formativo y de las necesarias relaciones que se establezcan entre ellas. El artículo presenta un acercamiento a esta problemática.

Palabras clave: diseño curricular; enseñanza de la informática; soberanía tecnológica.

Resumo: É analisada a concepção de soberania tecnológica para o tratamento dos sistemas a serem implantados no país e as decisões sobre sua modificação. No entanto, esse é um processo complexo para sua implementação, se o desenvolvimento de competências informacionais em professores de informática, com base em software livre, não for alcançado. A análise da formação desses profissionais deve partir da estruturação de um projeto curricular com disciplinas próprias e optativas que se estruturam de acordo com os objetivos do processo formativo e as relações necessárias que se estabelecem entre elas. $\mathrm{O}$ artigo apresenta uma abordagem para esse problema.

Palavras-chave: desenho curricular; ensino de informática; soberania tecnológica.

\begin{abstract}
The conception of technological sovereignty for the treatment of the systems to be implemented in the country and the decisions on their modification are analyzed. However, this is a complex process for its implementation, if the development of informational competencies in computer science teachers, based on free software, is not achieved. The analysis of the training of these professionals must start from the structuring of a curricular design with its own and optional subjects that are structured according to the objectives of the training process and the necessary relationships that are established between them. The article presents an approach to this problem.
\end{abstract}

Keywords: curricular design; computer education; technological sovereignty.

Para citar - (ABNT NBR 6023:2018)

HERNÁNDEZ, Walfredo González; REVILLA, Dayana Olivia Hernández; CASTILLO, Juan Manuel González. La migración hacia software libre en la formación de profesores de informática como estrategia de soberanía tecnológica. Eccos - Revista Científica, São Paulo, n. 58, p. 1-18, e17195, jul./set. 2021. Disponível em: https://doi.org/10.5585/eccos.n58.17195. 


\section{Introducción}

La concepción de soberanía en Cuba ha sido objeto de análisis desde diferentes aristas y por ejemplo en el marco político, tomó mayor sentido desde el inicio de las guerras de independencia del dominio colonial en el siglo XIX. Desde esa época comienza realmente el análisis de las cuestiones relacionadas con la soberanía de nuestro país, y con el decursar de la sociedad ha devenido como parte del análisis de la soberanía nacional, la concepción sobre la soberanía tecnológica. No es hasta décadas posteriores del siglo XX que se logra construir una plataforma tecnológica que permita la preparación de los recursos humanos necesarios y se consoliden las carreras relacionadas con los procesos de informatización. Sin embargo, la formación de profesores ha estado ajena a este proceso y estos profesionales constituyen el eslabón base de este proceso, quienes al desarrollar las competencias informacionales necesarias para la educación superior, serían los responsables de formar con los requerimientos necesarios a las nuevas generaciones.

Las carreras en la educación superior, desde la concepción cubana, tienen como uno de sus fines dar respuesta a las necesidades de especialistas en la sociedad, en especial a una sociedad que comienza un proceso de informatización a gran escala. En el caso de la Licenciatura en Educación, Especialidad Informática, surge por la necesidad de formar profesores de informática debido al auge de los procesos de informatización en nuestro país. Otra arista de la formación de estos profesionales está en la satisfacción de las necesidades cada vez más crecientes de la comunidad, a partir de la creciente informatización de la sociedad cubana. De este análisis se desprende la importancia que se le concede a los procesos de informatización y, sobre todo, a los procesos de formación en informática de la sociedad. Si se asume que la escuela es la organización generadora de la cultura en la comunidad, entonces el profesor de informática es el encargado de los procesos de informatización del barrio. Todo lo expresado pone de manifiesto la importancia de la formación de este profesional.

De esta manera, el profesor de informática no sólo está encargado de los procesos formativos en de esta rama del conocimiento en la escuela sino, de muchos de los procesos de informatización de la sociedad. De esta afirmación se desprende que es importante atender a las necesidades de informatización de la comunidad, además del desarrollo de una cultura informacional en su contexto. Sin embargo, la formación de un profesional con estas características parte de concebir un diseño curricular que permita este proceso de extensión de la escuela hacia la comunidad. Para ello el sistema educativo cubano establece un proyecto curricular que permite a los estudiantes poseer una preparación similar a partir de un currículo 
llamado base que es igual para todos en las diferentes universidades del país. En este currículo base pudiera expresarse una contradicción con lo local de la comunidad y sus necesidades puntuales de informatización. Sin embargo, también se incluyen en el proyecto las asignaturas propias, optativas y electivas que complementan la formación base a nivel nacional y les permite atender lo contextual. De esta afirmación se desprende la necesidad diseñar estas asignaturas en consonancia con las necesidades en la formación del profesional. Por consiguiente, el objetivo de este artículo es elaborar el diseño de las asignaturas propias y optativas para la formación del Licenciado en Educación Especialidad Informática de la provincia de Matanzas en Cuba sobre la base de la soberanía tecnológica.

\section{Desarrollo}

\section{El diseño curricular en la educación superior}

La formación de profesores de informática es una necesidad innegable de los procesos de informatización de cualquier sociedad donde los procesos de informatización social desempeñen un papel importante. De hecho, en la concepción cubana, su papel de alfabetizador digital es tan importante para la sociedad como lo es otra organización social denominada Joven Club de Computación y Electrónica (MONTERO e HERNÁNDEZ, 2015) que tiene un papel importante en la informatización social. Entre la escuela y el Joven Club debe existir coordinación de todas las acciones de informatización que se emprendan. Sin embargo, para este empeño social el profesor debe estar preparado para enfrentar las tres aristas de introducción de la informática en la educación. Una primera aborda la introducción de la informática en la enseñanza tanto como medio de enseñanza como de soporte para la gestión escolar. Una segunda se trata de la enseñanza de la informática y la tercera sobre el desarrollo de software educativo, ello implica que el profesional debe poseer competencias en las tres aristas ya mencionadas. Pero, para alcanzar la formación profesional necesaria en estas tres aristas, esta concepción debe incorporarse como parte del diseño curricular.

A partir de lo expresado es necesario adentrarse en qué concepción de diseño curricular debe tenerse en el proceso formativo. Son variadas las concepciones que sobre este proceso han permeado la educación, para un autor es“... el conjunto de criterios, planes de estudio, programas, metodologías y procesos que contribuyen a la formación integral y a la construcción de la identidad cultural nacional, regional y local, incluyendo también los recursos humanos, académicos y físicos para poner en práctica las políticas y llevar a cabo el proyecto educativo institucional" (ALDANA, 2006, p. 2). 
En este caso no se aborda con la integralidad necesaria al excluir los actores de este proceso de diseño, cuestión esta trascendental pues son los encargados, por un lado, de su elaboración y, por otro, de su implementación en la práctica.

Para otra autora el currículo es “... un proyecto educativo integral y no se refiere a un aspecto determinado del proceso educativo, además se evidencia que los diseños curriculares tienen que concebirse como una tarea de investigación con un carácter dinámico, donde los alumnos y maestros se impliquen con una posición afectiva por el conocimiento, para darle solución a los problemas y proyectarse hacia el futuro" (GARCELL, 2006, p. 12).

Se puede apreciar en esta definición el carácter activo y consciente de una parte de los implicados en el proceso de diseño curricular: los componentes del proceso, sin embargo, no se aborda así otros actores: que deben implicarse en el diseño curricular. Esto hace que se obvie uno de los elementos esenciales de este: su intencionalidad basada en las representaciones sociales de aquellos que lo concibieron para ser ejecutados por un conjunto de personas. Si se asume esta característica esencial de los currículos es necesario entonces definir quiénes son los encargados de elaborarlos.

Uno de los resultados teóricos actuales en el área de la enseñanza con un enfoque histórico cultural es la didáctica antropológica (CHEVALLARD, 1998). Para esta teoría didáctica es esencial el concepto de transposición didáctica, en la cual se expresa cómo se lleva del saber sabio al saber enseñado como lo expresa el propio autor Chevallard (1998), lo cual para la informática resulta un tema controversial.

Siguiendo el análisis de las definiciones de currículo, para otros autores se asume “... como la síntesis de elementos culturales de la institución. Implica los conocimientos, prácticas, valores, costumbres, hábitos, creencias, procedimientos, tendencias, que conforman una propuesta educativa. Esta es construida por la participación de diversos grupos y sectores que representan la diversidad con intereses, muchas veces contradictorios, donde algunos tienden a ser hegemónicos y otros a resistirse a esa hegemonía” (CANDREVA e SUSACASA, 2009, p. 12).

Esta definición de currículo incorpora un aspecto esencial en la actualidad: la existencia del poder y su expresión en los procesos educativos. Es insoslayable la presencia de los grupos de poder en la educación y su interpretación de las necesidades sociales que deben ser resueltas por esta. De esta manera puede suceder que los currículos sean la expresión de las necesidades de los grupos hegemónicos y no las necesidades de formación de la sociedad en general. Es por ello que es muy complejo elaborar currículos apolíticos.

De otra manera “... el currículo es un proyecto educativo global que asume un modelo 
didáctico conceptual y posee la estructura de su objeto: La enseñanza - aprendizaje. Tiene carácter de proceso que expresa una naturaleza dinámica al poseer su objeto relaciones interdependientes con el contexto histórico - social, la ciencia y los alumnos, condición que le permite adaptarse al desarrollo social, a las necesidades del estudiante y a los progresos de la ciencia” (ADDINE FERNÁNDEZ, 2010, p. 34).

En esta definición se aprecian algunos elementos que superan las concepciones anteriores entre las cuales se encuentran la naturaleza dinámica del mismo que logre la formación de los alumnos. En este sentido, es importante para la informática como ciencia, que el currículo sea dinámico y flexible que permita incorporar los avances de esta ciencia en el menor plazo posible. Ello se interpreta como la necesidad de una cualidad del currículo: su carácter proyectivo. La obsolescencia de los contenidos informáticos que se abordan en la escuela es una de las cuestiones de mayor importancia para la formación de este profesional. Una solución es basar el currículo en los núcleos conceptuales básicos y no básicos (HERNÁNDEZ, 2016b) en los cuales se expresa la esencia de la informática y sus líneas de desarrollo, lo que forma parte del enfoque de sistema propuesto por el mismo autor en artículos posteriores (GONZÁLEZ HERNÁNDEZ, 2019a).

A partir de los elementos expuestos por los diferentes autores consultados, se asume que el currículo es el resultado de un proceso de concepción de un proyecto sistémico derivado de la acción de variados agentes sociales, relacionados con el proceso educativo en el cual se entrelazan diversos componentes culturales, didácticos y pedagógicos para interactuar con la sociedad en el presente y con una proyección futura teniendo en cuenta el desarrollo social. Una vez clarificada la concepción teórica que se asume en este artículo, se puede pasar al análisis de la soberanía tecnológica, elemento indispensable para trabajar el diseño curricular del profesional de la informática.

\section{La migración a software libre como cuestión de soberanía tecnológica}

La soberanía tecnológica es uno de los principios fundamentales de la soberanía nacional, entendida esta, como la determinación de un país de la forma en que se relaciona con los demás y también de la manera que resuelve sus asuntos internos, se deriva de esto la necesidad de la independencia de los estados para poder ejercerla. De ahí que emanen varios conceptos de soberanía como la económica, la jurídica y, la que ocupa este artículo, la soberanía tecnológica. Para varios autores (DAUDINOT LÓPEZ e MILLER CLEMENTE, 2016) la soberanía tecnológica se relaciona con la posibilidad de no depender de ningún otro estado en 
términos de tecnologías. Una de las dependencias más conocidas es el pago de licencias por concepto de compra de software del llamado software propietario. Para Bordignon (2015) la soberanía tecnológica se define como “... la capacidad soberana de todo país en lo referido a planificar y llevar adelante aquellos proyectos propios que hagan uso intensivo de tecnología, ya sea de producción nacional como también extranjera, pero adaptada a sus propios intereses" (BORDIGNON, 2015, p. 79).

Para ello, el propio Bordignon (2015) define entonces, que un objetivo de todo país debe ser lograr un nivel adecuado de autonomía tecnológica, lo cual implica ejercer la capacidad de elección de aquello que se va a desarrollar y aquello que se importará. Lo ideal en este contexto sería asumir la soberanía tecnológica con la autosuficiencia a para el desarrollo y fabricación de todo con tecnología nacional. En este sentido es importante asumirlo como el sistema de criterios propios de la tecnología que más conviene, donde ésta puede ser nacional o importada. Dentro del dominio de la soberanía tecnológica, estas reflexiones se orientan hacia las tecnologías digitales, dado que apropiarse de ellas y de su espacio en el mundo es un buen camino para empezar a recorrer el camino de la independencia tecnológica. Con respecto a los individuos se entiende que ellos pueden ejercer su soberanía, cuando tienen saberes y habilidades que los hacen ser autosuficientes en el uso de las tecnologías digitales, teniendo libertad para disponer de la tecnología y usarla en la forma y condiciones que deseen.

Otro de los elementos reconocidos en la literatura con respecto a la soberanía tecnológica se centra en la infraestructura que sustente los procesos en las organizaciones. De la infraestructura es importante el análisis de dos cuestiones fundamentales, la primera se centra en el hardware y la segunda en los sistemas informáticos que se instalan. Con respecto al hardware es necesario reconocer la necesidad de comprar aquellos equipos que posean un hardware libre. Este concepto de hardware libre definido como “... cuyo diseño se pone a disposición del público de modo que cualquiera puede estudiar, modificar, distribuir, hacer y vender el diseño o el hardware que se sustente en dicho diseño [...] el diseño a partir del cual se construye está disponible en el formato que se prefiera para que se hagan modificaciones en él. [...] Idealmente, el hardware libre utiliza elementos y materiales inmediatamente disponibles, procesos estandarizados, infraestructura abierta, contenido no restringido y herramientas de diseño libres para aumentar al máximo la capacidad de los individuos de hacer y utilizar el hardware. El hardware libre brinda a los individuos la libertad de controlar su tecnología mientras comparten el conocimiento y alientan el comercio a través del intercambio abierto de diseños”. (LAZALDE et al., 2014, p. 4)

Esta definición permite asumir que la utilización intensiva de equipamiento con 
hardware libre, presupone la modificación de sus componentes de manera eficaz reduciendo las curvas de aprendizaje o eliminando la ingeniería inversa. De la misma manera pueden ser optimizados los códigos ya realizados en el kernel del sistema operativo para manejar de manera más eficiente los diferentes periféricos o componentes de la computadora.

El otro elemento se entronca con este anterior es el uso intensivo también de sistemas informáticos libres. La libertad en los software es algo que ha sido discutido ampliamente en la literatura (DAUDINOT LÓPEZ e MILLER CLEMENTE, 2016; RASHID et al., 2019) y es necesario abordar sobre todo para la formación universitaria. Todos los autores ya mencionados enfocan la necesidad de la formación profesional utilizando software libre por dos cuestiones fundamentales. En un primer lugar es importante para lograr independizar los procesos formativos universitarios del software propietario, portadores de puertas traseras y filosofías no acordes a nuestros modelos de desarrollo y cultura. Una segunda cuestión se encuentra relacionado con las necesidades que tendrán estos egresados de utilizar diferentes software en los procesos en los cuales participan una vez que sean ubicados en las diferentes organizaciones. Se asume que un estudiante formado en esta filosofía puede llevarla a las organizaciones en la cuales se encuentra y sustituir las soluciones propietarias que se encuentren en su área de actuación. Para ello es necesario que desde los currículos en las enseñanzas media y básica se trabaje también utilizando estos software.

De los elementos planteados se puede asumir entonces que la migración hacia software libre es una cuestión de soberanía tecnológica. El ser autosuficientes en el uso de las tecnologías digitales implica modificarlas y cambiarlas a partir de las necesidades y decisiones que haya tomado el país respecto a éstas. Esto entraña la necesidad de formar a los recursos humanos necesarios para enfrentar esta tarea. Uno de los recursos humanos necesarios para la migración hacia el software libre lo constituyen los profesionales de la educación. Estos profesionales son los encargados del desarrollo de las competencias informacionales en la enseñanza media y lograrlo en un entorno libre. De ahí la importancia de la formación de estos profesionales en un entorno de este tipo.

Como bien se plantea "El protagonismo de las TIC en la llamada sociedad de la información subraya la pertinencia de incluir los derechos digitales en el acervo reivindicativo de los movimientos sociales, máxime cuando se ponen en evidencia las implicaciones políticas de unas tecnologías que por su carácter comunicativo afectan directamente a los procesos sociales y políticos" (CANDÓN-MENA, 2012, p.76). La integración de la tecnología a los diferentes entornos sociales ha llevado a procesos que hasta ahora no eran importantes. El análisis de los acontecimientos del 11-M permiten avizorar las potencialidades que poseen estas 
tecnologías en la movilización de las personas. Cuando el uso de estas tecnologías se realice para todos los ámbitos de la vida y se realice de manera extensiva e intensiva es importante el análisis del impacto que tienen en las actividades humanas.

En este análisis es importante tener en cuenta desde una perspectiva psicológica el encuentro que se produce entre personas de culturas diferentes, los símbolos y signos diferentes que pueden aprender entre ellos. Este proceso de intercambio de culturas que se produce en la época actual sobre la base de las tecnologías transcurre con una velocidad impresionante. Quiere decir entonces que se puede aprender y asumir posturas propias de otras culturas y su traslado a contextos totalmente diferentes, lo cual puede llevar a conductas enajenantes para el grupo social en el cual se encuentran. Ello puede conducir a un análisis negativo de las tecnologías cuando ese no es su papel, aunque cumplan con una de las características actuales de las tecnologías: la extensibilidad de sus valores de uso. Un ejemplo de ello se encuentra en la computadora que surge para cálculos numéricos y actualmente potencia el entretenimiento de los seres humanos aun cuando conserva su uso original.

De hecho, en la misma medida que las tecnologías se integran a las más disímiles actividades humanas así será el dominio que tengan a partir de la filosofía que traen consigo. Así será también la dependencia de los grupos humanos de estas tecnologías, cuestión esta de especial importancia en la actualidad en un proceso real de guerras culturales (DOS SANTOS, 2020). Las ideas esenciales de dominación desde las tecnologías que se realiza en el mundo actual están bien claras y se expresan desde varias aristas.

El análisis de los procesos de dominación cultural a través de las tecnologías que están presentes en la sociedad actual es de vital importancia. Un aspecto importante en los procesos de dominación cultural está en el conocimiento de los patrones de conducta e intereses de los grupos humanos en estudio. De ahí se trazan estrategias de dominación encaminadas a integrarlos a los patrones culturales que interesan a los centros de poder. Otro aspecto importante del análisis está en la obtención de la información de los perfiles de las personas y la comunicación que establecen con otras personas. De ahí que la obtención de la información necesaria para la dominación de los grupos humanos sea muchas veces a través de programas soportados en tecnologías o de puertas traseras de los propios sistemas propietarios. Esta es otra arista, de la necesidad urgente de la mudanza de nuestras tecnologías hacia el movimiento libre.

De ahí que se considere en este artículo que el uso de tecnologías libres es una condición necesaria para el logro de la soberanía tecnológica, sin embargo no es la única. Es primordial para el logro de la soberanía tecnológica la preparación de la sociedad para el uso de estas tecnologías libres. Por ello, que la extensión de esta filosofía libre a toda la sociedad y a todas 
sus actividades, argumenta la necesidad de políticas orientadas a la informatización de los procesos sociales con estas herramientas. Una arista de estos análisis y que garantizan la continuidad de estos procesos es la formación de profesores de informática donde se utilice y se demuestre la importancia del movimiento libre. Una posible solución es la inclusión en el currículo de la formación de estos profesionales de las herramientas libres.

De este acápite se puede inferir la necesidad del uso de tecnologías libres es una necesidad insoslayable para los países que han decidido apartarse de los caminos dictados. Para ello una de las vías de solución es la formación de profesionales utilizando las tecnologías libres y en el caso de los profesores es aún más importante. En el caso de los profesores de informática este análisis es esencial pues ellos serán entes informatizadores de la comunidad donde está enclavada la escuela donde laboran.

\section{La informática como ciencia y su reflejo en el currículo de la formación de profesores}

La informática es una de las ramas del conocimiento humano que más rápido se desarrolla en la actualidad por lo que los contenidos universitarios enfrentan una rápida obsolescencia. Esta ciencia en su desarrollo histórico se ha preocupado por los procesos de captación, procesamiento, protección y transmisión de la información lo cual la ha llevado a modelar estos procesos de la realidad (EXPÓSITO RICARDO, 2009). Otra característica de la informática, es la diversidad de software que existen para diferentes actividades como expresión del nivel de penetración alcanzado en otras ciencias. Sin embargo, una de las características fundamentales de ella es el rápido crecimiento de sus principales disciplinas, el nivel de desarrollo que han presentado en la actualidad y, sobre todo, el alto impacto que han tenido en el desarrollo de la sociedad actual.

Una de las líneas de desarrollo que marca la informática en la actualidad es la interrelación entre dos elementos que se interrelacionan entre sí: hardware y software. Este par ha marcado el resto de las líneas de desarrollo de la informática y marcó pautas en el desarrollo de la Ingeniería del Software con la conocida crisis del software. A partir de este momento se tomó consciencia de la necesidad del desarrollo de métodos sistemáticos para el desarrollo del software. En el mismo orden de ideas, otros dos elementos interrelacionados entre sí marcan otras líneas de desarrollo: la ingeniería del software y la programación. Cada una de ellas han continuado de manera acelerada su desarrollo, sin embargo, la ingeniería del software contiene a la programación como una de las etapas del proceso de desarrollo. Para el autor de este artículo la ingeniería del software marca el camino a seguir y la programación marca el cómo se transita 
por ese camino.

De otra manera se encuentran varias de las vertientes de desarrollo orientadas al almacenamiento de los datos: las bases de datos y, en su concepción más actual, los almacenes de datos. El almacenamiento de grandes volúmenes de datos y su posterior procesamiento han llevado a que los datos hoy no se almacenen en el orden de los TB al mismo tiempo que no se procesan por una única computadora y sí por sistemas distribuidos que fusionan las capacidades de varias máquinas en un procesamiento único. Esto ha conducido al surgimiento desde hace algunos años de lo que se conoce por computación distribuida y se ha llevado a actividades humanas que hasta hace poco estaban alejadas de la informática como, por ejemplo, el arte.

Otras de las vertientes de la informática que en la actualidad ha tenido un auge significativo se encuentra en las redes y más aún en la conectividad de diversos periféricos a internet. Lo que se ha dado en llamar "la internet de las cosas" para designar una amplia variedad de periféricos conectados entre sí por los más disímiles canales de comunicación entre los que se destacan las conexiones inalámbricas (YANG et al., 2017; YAQOOB et al., 2017). Esto hace que dentro del sistema de carreras en la educación superior sean las relacionadas estas conexiones, la de mayor obsolescencia por lo cual deben implementarse cursos relacionados con estas vertientes de desarrollo de la informática. Otro elemento a tener en cuenta además del expuesto son las necesidades de la entidad empleadora: la escuela.

Hoy está definido por el Ministerio de Educación en Cuba (MINED) que la escuela está llamada a constituirse como el ente cultural más importante de la comunidad. En el contexto de los profesores de informática éstos deben contribuir con los procesos de informatización social y pueden lograrlo, a partir de la alfabetización digital de su comunidad. Sin embargo, esto no es posible hoy en el contexto cubano sin hablar de Internet, tecnologías móviles y telefonía entre otras cuestiones. Por lo que también deben ser incluidos en su formación como profesores de informática estas cuestiones, aunque hay que prestar atención a que estas necesidades pueden variar con el tiempo. Para resolver estas problemáticas curriculares abordadas en este párrafo es importante diseñar un currículo que pueda ser rediseñado y que, al mismo tiempo, mantenga aquellas disciplinas que son esenciales en su formación. Una solución es crear un currículo con asignaturas base, que constituyen los elementos esenciales que no deben ser soslayados en su formación; las asignaturas propias, que responden a necesidades del territorio y que tienen determinada importancia dentro de su formación y, por último, aquellas asignaturas optativas que responden a las necesidades que pueden ser variadas con el decursar del proceso formativo.

Después de un análisis de las asignaturas base de esta carrera y los elementos que se han expuesto hasta el momento se realiza una propuesta de asignaturas propias desglozadas por año. 
En el primer año de la carrera se incluyen dos asignaturas distribuidas una por cada semestre. Se comienza con la asignatura denominada introducción a la informática en la cual se explican las líneas de desarrollo de la informática, los procesos de informatización de las organizaciones y sus principales disciplinas científicas. Ya en el segundo semestre se les enseñan los sistemas operativos libres haciendo énfasis en Edubuntu, una distribución de Ubuntu que provee herramientas para el desarrollo de aplicaciones educativas en este sistema operativo. Esta elección del sistema operativo se basa en la utilización posteriormente de sus herramientas para el desarrollo de software educativo en un ambiente libre, con ello se contribuye a su efectividad pues ya es conocido previamente por el estudiante. Esto lo logra a partir de la incorporación de GCompris y la KDE Edutainment Suite.

De la misma manera que se definen las asignaturas propias también se determina las optativas, cuya peculiaridad es que se deben ofertar varias para que el estudiante seleccione una de ellas, pues coexisten en el mismo horario. En el caso de las optativas se sigue la idea de ofertar grupos de asignaturas que respondan a las tres vertientes de introducir la informática en la enseñanza ya explicitada con anterioridad. Esto permite que el estudiante no pueda sortear una de estas vertientes y se prepare en las tres, de tal manera que pueda cumplir su misión de ente informatizador de la comunidad en la cual esté situada la escuela. Estas optativas, al ser complementos de formación base, dependen de un conjunto de asignaturas que deben ser impartidas con anterioridad por lo cual no pueden comenzar desde el primer año de la carrera. A partir de este principio, es que se comienza el análisis de cuál grupo de asignaturas colocar por año. Las relacionadas con la enseñanza de la informática deben comenzar a partir del 3 año puesto que este es el año en el cual se concluye la formación pedagógica de los estudiantes con la asignatura Didáctica de la Informática. después de haber transitado por asignaturas como Psicología, Pedagogía y Didáctica en ese orden en la malla curricular.

A partir de este mismo análisis se ubica el grupo de asignaturas de introducción de software educativo en la enseñanza, dado que el nivel de preparación de los estudiantes para este propósito no es el más adecuado. De este análisis se deriva que los procesos de desarrollo de software educativo pudieran ser candidatos para constituir el primer grupo de estas asignaturas. De ellas se desprenden dos tecnologías para la educación que pueden ser usadas en la escuela: el video educativo y el software educativo, aunque este último puede contener al primero. En el análisis del video educativo hay dos momentos para su realización, uno el momento del diseño y otro el de edición. Al concretarse estas actividades en roles que se desempeñan en un proyecto de video pueden realizarlos en grupos donde intervengan estudiantes de ambas asignaturas e integrarse en la evaluación usando para ello el aprendizaje 
basado en proyectos (GONZÁLEZ-HERNÁNDEZ e COLOMA-CARRASCO, 2018; GONZÁLEZ HERNÁNDEZ, 2018; 2019b; GONZÁLEZ-HERNÁNDEZ, 2016c). Para ser consecuentes con estos análisis es preferible estudiar las herramientas de desarrollo para la creación de mundos inmersivos y las herramientas de desarrollo de software educativo, que facilite su selección de conjunto con las metodologías en años posteriores. Para ello EDUBUNTU provee de un conjunto de herramientas importantes.

Por las características de esta distribución se considera importante dedicar un espacio a su análisis en este artículo. Esta distribución de Ubuntu está creada para ofrecer a los profesores herramientas para la creación de software educativo, además de proveer diversos software educativos para los niveles de enseñanza. En la instalación del sistema operativo con un peso de $4 \mathrm{~Gb}$ se pueden encontrar software educativos pensados desde los 3 años de edad hasta los 18 años. Además, traen incluidos paquetes para la enseñanza de la Matemática basados en la idea expresada en una abundante bibliografía (PÁSZTOR et al., 2015; PO-SHENG et al., 2017; GARCÍA VÁZQUEZ et al., 2018; SIMON et al., 2018) acerca de la utilidad de las tecnologías para la enseñanza de las Matemáticas. Otra de las herramientas que provee ampliamente para el aprendizaje se encuentra en los juegos educativos que en la literatura se ha denominado serious game (MARTÍN, 2008; PALOMINO TAYPE, 2016; JERČIĆ e SUNDSTEDT, 2019; STANITSAS et al., 2019; LIUA e LIU, 2020). Quiere decir que este sistema operativo, desde su propio DVD de instalación disponible en internet, provee de una amplia variedad de software educativos para los estudiantes de nivel medio. Otra característica de este sistema operativo está dada por la no violencia presente en sus software educativos, cuestión esta preocupante en varias propuestas propietarias. Esta característica lo hace un sistema operativo ideal para la enseñanza y por ello necesario para la formación de los profesores de informática.

Ya para el año final quedan otras asignaturas que les permiten enfrentar la asesoría a otros profesores para la introducción de la informática como herramienta de trabajo y para desarrollar sus propias herramientas por parte de otros profesores de la escuela. Esto explica que ellos deben apropiarse de las estrategias de informatización, así como las herramientas más comunes para lograrlo. En este propósito de soporte educativo se inscriben los EVEAS que les brindan plataformas interactivas en las cuales se puede insertar los cursos de los profesores. Sin embargo, para ello es necesario que conozcan cómo se administran los servidores en el entorno escolar para la instalación de estos EVEAS y que sean capaces de comprender las actuales tendencias de adaptabilidad de éstos en sus procesos de interacción con los estudiantes.

En el último semestre, se cierra el proceso de formación para el desarrollo de aplicaciones educativas con el desarrollo de sistemas para móviles. Esta es una tendencia en la 
cual se debe insertar la escuela cubana dado la gran cantidad de estudiantes que poseen móviles a estas edades. En cuanto los estudiantes se gradúen, 4 años después, ya el aprendizaje ubicuo es una realidad que debe ser potenciada y que está en consonancia con los procesos de informatización social a gran escala que se están viviendo a nivel mundial. Este tipo de aprendizaje cambia totalmente el paradigma de aprendizaje que se viene dando desde la antigüedad: el conocimiento está en los centros del saber y para acceder debo ir hasta ellos. El cambio paradigmático está dado en que el saber se acerca a la persona y está disponible en cualquier momento sin importar el lugar donde se encuentre. Para ello la infraestructura tecnológica es determinante.

En esta asignatura también se utilizan como software libre Android con su SDK y el entorno de desarrollo (IDE en inglés) que han utilizado en la carrera que es eclipse. El eclipse es un ambiente de desarrollo que permite programar en el lenguaje java totalmente libre. Este IDE provee de las herramientas necesarias para codificar los programas realizados por los estudiantes para el entorno Android, uno de los más utilizados a nivel mundial (AMALFITANO et al., 2019; PATRICIA VILLAREAL-FREIRE et al., 2019). Eclipse plantea una filosofía muy cómoda de desarrollo basado en plugins. El plugins es un paquete de software que se puede incorporar en dependencia de las necesidades que se posea como desarrollador y posteriormente retirar cuando haya terminado de utilizarlo. Una de las ventajas fácilmente detectable de esta filosofía está en el ahorro del espacio en memoria cada vez que se ejecute el IDE. De ahí la importancia que tiene para computadoras escolares de prestaciones media como las que se encuentran en las escuelas.

De esta manera se forma a un estudiante para atender las tres vertientes de la introducción de la informática en la escuela. Este diseño de sistema de asignaturas responde a una idea básica de los autores (HERNÁNDEZ et al., 2004; GONZÁLEZ HERNÁNDEZ et al., 2006; GONZÁLEZ HERNÁNDEZ, 2015; HERNÁNDEZ, 2016b) que trata la integración de dos enfoques conocidos: el enfoque problémico y el enfoque de proyecto (EXPÓSITO RICARDO, 2009) al cual fueron variadas sus condiciones iniciales, para su integración y por otro lado el enfoque de sistema (GONZÁLEZ HERNÁNDEZ et al., 2006) que plantea como construcciones teóricas fundamentales: el núcleo conceptual y las líneas directrices.

En la concepción cubana de la educación superior el estudiante debe estar vinculado a diferentes centros laborales con objetivos a cumplir por años que les permita adquirir las competencias necesarias para su desempeño laboral. En este tránsito por las escuelas, en el caso que ocupa a esta propuesta, los estudiantes deben detectar y solucionar determinadas problemáticas escolares. En la opinión de este autor, estas problemáticas deben estar 
relacionadas con la inserción de la informática en el centro, en consonancia con las tres vertientes ya enunciadas en párrafos anteriores. Para ello, se dispone de un fondo de tiempo en la malla curricular y en la planificación docente de cada semestre. Se concibe que esta práctica laboral transcurra como un proceso de formación gradual y que se complejice a medida que avance la formación de los estudiantes. En esta práctica es donde van a integrar los componentes cognitivos, afectivos, metacognitivos y sociales que les permitan desarrollar las competencias profesionales. Una de ellas es el desarrollo de aplicaciones educativas utilizando software libre.

También es importante destacar que otro de los procesos sustantivos de la Educación Superior está representado en esta malla curricular, es la investigación considerada la vía esencial para la resolución de los problemas que ellos pueden encontrar en su práctica profesional. Pero este propósito no se alcanza si no se sistematiza y se incorpora a sus modos de actuación de manera permanente. Para ello se van desarrollando desde los primeros años, acciones investigativas que integren las asignaturas que reciben como parte del componente académico con los problemas profesionales que detectan en la práctica laboral. Por otra parte, se integran a este proceso los métodos de investigación que irán asimilando en la medida que avanza su formación. De esta manera los estudiantes deben entregar informes de práctica laboral en los cuales se recogen tanto los problemas que detectaron, los análisis que han sido realizados ante problemas similares y la solución que ellos proponen ajustada al contexto escolar en el cual se desenvuelven.

Estos problemas pueden constituir la base de investigaciones de mayor alcance conducentes a un trabajo de curso en el cual fundamenten con mayor rigor la solución a la problemática detectada. Este trabajo de curso ya posee una estructura fijada por el Colectivo de Carrera y ellos deben ajustarla. Se trata de un documento en el cual reflejen los pasos esenciales de solución de la problemática aplicando los resultados de su formación pedagógica y los aspectos esenciales de la metodología de la investigación. De esta manera se van perfilando los elementos esenciales acerca de los fundamentos teóricos de la propuesta y en la Tesis de Diploma, ejercicio de culminación de estudios, se tiene ya hasta la validación de la propuesta.

\section{Conclusiones}

La formación informática de los profesionales de esta ciencia lleva a tener en cuenta la obsolescencia de los contenidos en su planeación para incorporar en sus modos de actuación los aspectos esenciales de esta ciencia y que lleguen a las escuelas para cumplir la encomienda de ser entes informatizadores de la comunidad. La solución planteada en este artículo está en la 
concepción de un currículo con asignaturas propias y optativas que permitan incorporar los avances de esta ciencia y transformar la comunidad.

La integración de varias asignaturas optativas y propias deben responder a la precedencia entre ellas, a su integración con el resto del currículo base, así como con el resto de los componentes investigativo y laboral. La imbricación de estos tres componentes en un sistema armónico que propicie los elementos esenciales de su formación y que cumpla con los objetivos del profesional es una de las cualidades de la propuesta desarrollada.

\section{Referencias}

ADDINE FERNÁNDEZ, F. La didáctica general y su enseñanza en la Educación Superior Pedagógica. Aportes e impacto. (Doctor en Ciencias), Cuba: Universidad de las Ciencias Pedagógicas Enrique José Varona, 2010.

ALDANA, I. A. M. Currículo de educación formal. Currículo de educación formal de niños y jóvenes, v. 2, n. 3, p. 34-42, 2006.

AMALFITANO, D. et al. Combining Automated GUI Exploration of Android apps with Capture and Replay through Machine Learning. Information and Software Technology, v. 105, p. 95-116, 2019.

BORDIGNON, F. R. A. Soberanía tecnológica y educación: una dupla indisoluble. Prólogos, v. VII, p. 79-102, 2015.

CANDÓN-MENA, J. I. Soberanía tecnológica en la era de las redes. Revista internacional de pensamiento político, v. 7, 73-92, 2012.

CANDREVA, A.; SUSACASA, S. Diseño curricular por competencias. Educación Médica Permanente, v. I, n. 2, , p. 78-89, 2009.

CHEVALLARD, Y. La transposición didáctica. Del saber sabio al saber enseñado. Francia: AIQUE, 1998.

DAUDINOT LÓPEZ, M.; MILLER CLEMENTE, R. A. Una solución pacs cubana bajo software libre que sirve de plataforma a especializaciones médicas. Revista Cubana de Informática Médica, v. 8, n. 2, p. 186-196, 2016.

DOS SANTOS, F. R. C. O que se entende por Retórica da Guerra Cultural. Domínios de Lingu@gem,p.1-48, 2020.

EXPÓSITO RICARDO, C. Elementos de Metodología de la Enseñanza de la Informática. Ciudad de la Habana: Editorial Pueblo y Educación, 2009.

GARCELL, C. M. O. El currículo: Diseño, desarrollo y evaluación en la educación superior. Cuba: Editorial Universidad de Holguín, 2006. 
GARCÍA VÁZQUEZ, J. P. et al. Instructional strategies and information technologies used for supporting the undergraduate mathematics teaching process: Scoping review protocol. International Journal of Educational Research and Innovation (IJERI), v. 90, p. 27-31, 2018.

GONZÁLEZ-HERNÁNDEZ, W.; COLOMA-CARRASCO, Á. L. Estado actual de la competencia modelar en la formación del profesional informático de la Universidad de Matanzas, Cuba. Paideia, n. 60, p. 105-124, 2018.

GONZÁLEZ HERNÁNDEZ, W. Apuntes sobre Didáctica de la Informática. Ciudad de la Habana: Editorial Universitaria, 2015.

GONZÁLEZ HERNÁNDEZ, W. La intuición informática: estado actual en la carrera de ingeniería informática de la Universidad de Matanzas. EccoS - Revista Científica, n. 46, p. 191-213, 2018.

GONZÁLEZ HERNÁNDEZ, W. Definición del aprendizaje desarrollador de la informática por el profesional informático. Revista Educación en Ingeniería, v. 14, n. 27, p. 106-115, 2019a.

GONZÁLEZ HERNÁNDEZ, W. La integración de enfoques de enseñanza como vía para elevar la Didáctica en estudiantes de Ingeniería Industrial. Cuestiones Pedagógicas. Revista de Ciencias de la Educación, n. 27, p. 113-126, $2019 \mathrm{~b}$.

GONZÁLEZ HERNÁNDEZ, W.; ESTRADA SENTÍ, V.; MARTÍNEZ LLANTADA, M. El enfoque de sistema en la enseñanza de la Informática para el desarrollo de la creatividad Revista Enseñanza Universitaria, v. 32, p. 45 - 56, 2006.

GONZÁLEZ HERNÁNDEZ, W. La intuición informática: un acercamiento a su estudio. Revista Ingeniería, Matemáticas y Ciencias de la Información, v. 3, n. 5, 2016 a.

GONZÁLEZ HERNÁNDEZ, W. Propuesta metodológica para el tratamiento de conceptos y definiciones informáticos. Revista Electrónica Formación y Calidad Educativa, v. 4, n. 2, p. 45-62, $2016 b$.

GONZÁLEZ-HERNÁNDEZ, W. El diseño curricular de las asignaturas propias y optativas en la carrera Licenciatura en Educación Especialidad Informática. Revista Publicando, v. 3, n. 9, p. 196-217, 2016c.

HERNÁNDEZ, W. G.; ESTRADA SENTÍ, V.; MARTÍNEZ LLANTADA, M. Contribución al desarrollo de la creatividad a través de la enseñanza de la programación. Revista Pedagogía Universitaria, v. 9, p. 30-45, 2004.

JERČIĆ, P.; SUNDSTEDT, V. Practicing emotion-regulation through biofeedback on the decision-making performance in the context of serious games: A systematic review. Entertainment Computing, v. 29, p. 75-86, 2019.

LAZALDE, A.; TORRES, J.; VILA-VIÑAS, D. Hardware libre. Recomendaciones para el fomento de la innovación ciudadana. Secretaría Nacional de Educación Superior, Ciencia, Tecnología e Innovación y el Instituto de Altos Estudios Nacionales del Ecuador. Ecuador. 2014 
LIUA, S.; LIU, M. The impact of learner metacognition and goal orientation on problem solving in a serious game environment. Computers in Human Behavior, v. 102, p. 151-165, 2020.

MARTÍN, M. A. G. Arquitectura y metodología para el desarrollo de sistemas educativos basados en videojuegos. Madrid: Departamento de Informática, Universidad Complutense de Madrid, 2008

MONTERO, J. S.; HERNÁNDEZ, W. G. La habilidad modelar multimedia en los procesos formativos de los joven club de computación la habilidad modelar multimedia en los joven club de computación.Didasc@lia: Didáctica y Educación., v. VI, n. 2, p. 29 - 44, 2015.

PALOMINO TAYPE, I. Desarrollo de un videojuego casual multiplataforma para reducir el estrés. (Tesis de Pregrado). Facultad de Ingeniería, Escuela Profesional de Ingeniería de Sistemas, Universidad Nacional José María Arguedas, Andahuaylas - Apurímac. Perú, 2016.

PÁSZTOR, A.; MOLNÁR, G.; CSAPÓ, B. Technology-based assessment of creativity in educational context: the case of divergent thinking and its relation to mathematical achievement. Thinking Skills and Creativity, v. 18, p. 32-42, 2015.

PATRICIA VILLAREAL-FREIRE, A.; FELIPE AGUIRRE AGUIRRE, A.; ALBERTO COLLAZOS ORDOÑEZ, C. Reverse engineering for the design patterns extraction of android mobile applications for attention deficit disorder. Computer Standards \& Interfaces, v. 61, p. 147-153, 2019.

PO-SHENG, H. et al. The relative influence of domain knowledge and domain-general divergent thinking on scientific creativity and mathematical creativity. Thinking Skills and Creativity, v. 25, p. 1-9, 2017.

RASHID, M.; CLARKE, P. M.; O'CONNOR, R. V. A systematic examination of knowledge loss in open source software projects. International Journal of Information Management, v. 46, p. 104-123, 2019.

SIMON, M. A. et al. Towards an integrated theory of mathematics conceptual learning and instructional design: The Learning Through Activity theoretical framework. Journal of Mathematical Behavior, v. 52, p. 95-112, 2018.

STANITSAS, M.; KIRYTOPOULOS, K.; VAREILLES, E. Facilitating sustainability transition through serious games: A systematic literature review. Journal of Cleaner Production, v. 208, p. 924-936, 2019.

YANG, J. et al. Multimedia cloud transmission and storage system based on internet of things. Multimed Tools Appl, v. 76, p. 17735-17750, 2017.

YAQOOB, I. et al. Equirements, and open challenges internet of things architecture: Recent advances, taxonomy. IEEE Wireless Communications, v. 4, p. 1-16, 2017. 